\title{
Electrical power equipment digital twins. Basic principles and technical requirements
}

\author{
Leonid Darian ${ }^{1, *}$, and Leonid Kontorovych ${ }^{2}$ \\ ${ }^{1}$ Technical Inspection UES, JSC, 109074 Moscow, Russia \\ ${ }^{2}$ Energy Equipment Engineering, LLC, 70417 Zaporozhye, Ukraine
}

\begin{abstract}
The increasing complexity of the problems of asset management and ensuring the operational reliability of electric power equipment, which have to be taken into account by specialists at all stages of the life cycle, leads to the need to develop and implement digital twins. Based on the analysis of these problems, this work describes principles and technical requirements for the development of digital twins of electrical power equipment. The basic principles of developing the architecture of digital twins in the form of images, examinations, and functions of their components are stated. The general technical requirements for digital twins of electric power equipment are proposed. The functions of digital twin users at all stages of the life cycle of electric power equipment are given.
\end{abstract}

\section{Introduction}

For many industries, including the electric power industry, it has recently become increasingly important to solve the following problems:

- an objective complication of the tasks to ensure the operational reliability of electrical equipment, including high-voltage one, due to the significant changes that have been taking place in the electric power industry for the past few decades, e.g., the aging of assets and the increased risk of their failure. Meanwhile, the requirements of electrical energy consumers for a reliable supply remain at a high level. Therefore, to maintain the equipment that has worked out its normalized resource in proper condition, either an increase in operating costs or its replacement with the new equipment is required, which in the current economic conditions is a challenging task;

- optimal asset management due to the need to quickly make difficult decisions to ensure safe loading capacity of equipment, extend or reduce its service life, plan equipment outage for preventive and repair work;

- a lack of the unified diagnostic Center with improved condition models for collecting and processing operational data and issuing recommendations for further actions to the operating organizations;

- a lack of certified specialists and incentives to provide correct information about the technical condition of the equipment by the organizations operating it;

- an insufficient level of automation, which led to a sharp rise in the amount of complex information while reducing the completeness and reliability of the analysis of equipment failures causes due to the insufficient qualification of personnel operating microprocessor- based diagnostic systems and devices and a decrease in the number of highly qualified experts;

- a need for a quick analysis of big data of technical characteristics of electrical equipment installed at power facilities because of the rapid development of information technologies - digitalization of all fields, including the electric power industry.

One of the most effective and modern ways to solve the problem of optimizing asset management and ensuring the operational reliability of equipment without additional operating costs is the development and implementation of digital twins (DT).

Examples of basic concepts, requirements, and specific implementations of industrial DTs, including DTs of power equipment, are presented in [1-8]. However, these publications contain no information on the basic principles and technical requirements for the development of DTs of station and substation equipment. Therefore, the purpose of this work is to describe the principles and technical requirements for the development of DTs of electrical power equipment.

\section{Terms and Definitions}

The terms and definitions regarding DTs are currently not standardized, so we have to pay special attention to them to provide an unambiguous understanding of their meanings. It was not for nothing that the great mathematician and philosopher Descartes said: "People would get rid of half of their troubles if they could agree on the meaning of words."

The $D T[2,5]$ is a digital copy of a physical object or process that helps optimize business performance.

The DT of the equipment $[1,4]$ is a virtual model that either describes a real object at micro and macro levels

\footnotetext{
* Corresponding author: leonid.darian@g,gmail.com
} 
(acting as a duplicate of the finished specific product), or serves as a prototype of the future object. Moreover, any information that can be obtained during the testing of a physical object must also be obtained based on the testing of its DT.

There are three types of DTs: Digital Twin Prototype (DTP), Digital Twin Instance (DTI), and Digital Twin Aggregate (DTA) [5, 6].

The DTP is a prototype of the physical object that it characterizes. The DTP contains the information needed to describe and create a physical version of the object. This information includes manufacturing requirements, detailed $3 \mathrm{D}$ or 2D (datasheets or drawings) geometric models, specifications of materials, technological processes, technical requirements for procurement, and logistics of accessories. The DTP is used at all stages of production but does not interact with the physical object, and it corresponds not to the operating conditions but the test conditions at the manufacturing factory. Such properties are inherent in design subsystems with the ability to digitally simulate factory tests provided by the equipment standards.

The DTI describes a specific physical object with which the twin remains connected throughout its life cycle. Twins of this type usually contain:

- a simplified 3D model of the main structural elements with geometrical dimensions (i.e., the design of a physical object is taken into account approximately, mainly as a "black box" model);

- a specification for the materials used;

- a specification for processes with a list of operations that were performed when creating a physical object, as well as the results of various tests of the object;

- records of service assistance, including replacement of equipment units and operating indicators;

- measurement results obtained from sensors and instruments.

DTIs are used at all operation stages - from installation to disposal. The functions specified in DTIs are partially performed by diagnostic monitoring systems that collect, store and process signals from sensors, instruments, measuring and protective devices installed on the equipment and, according to specific algorithms, warn about the risks of equipment failures under the current operating conditions. Such systems do not allow predicting the equipment technical condition when the operating conditions change: the aging of materials, environmental changes, the occurrence and/or development of structural defects.

The DTA is a system in which the DTI and DTP are subsystems and exchange data with each other using random or proactive requests. At the same time, DTAs can use DTPs in full, simulating an arbitrary change in the geometry of the object, replacing its components, units, and structural parts with others, or use DTPs partially to predict changes in the technical condition when changing the impact on the object and/or the properties of the materials used. In this case, a change in the geometry and design is only partially permissible for certain predetermined cases associated with the occurrence and development of defects.

Additionally, we introduce the following definitions:

DT diagnostic criteria are physicochemical or calculated parameters of the DT, the nominal and permissible values of which fully characterize both the technical condition of the corresponding physical object as a whole and its single units for all modes (e.g., the criteria for electrical, electrodynamic, mechanical strength and stability of the equipment).

DT efficiency criteria are geometrical, physicochemical, or calculated parameters of the DT, the nominal and permissible values of which fully characterize the efficiency of functioning of the corresponding physical object as a whole and its components and units for all modes (e.g., the number of electricity losses in equipment, load capacity and equipment service life).

DT informative parameters are geometrical, physicochemical, or calculated parameters of the physical object transmitted to the DT, a change of which affects the corresponding diagnostic or performance criteria (e.g., ambient temperature and humidity, voltages and currents, external mechanical influence).

DT information models are a set of data, methods, algorithms, and programs for their input, storage, and visualization.(e.g., data on the design and functional parameters of the DT physical object, data on the conditions and manufacturing, installation and operation technology, data on preventive and repair works, etc.).

DT mathematical models are a set of techniques, algorithms, and programs based on the numerical or analytical solution of systems of equations (algebraic, ordinary differential, or with partial derivatives), as well as data on the properties of materials and external influences, simulating various multi-physical (e.g., electrical, electromagnetic, thermal, mechanical, etc.) steady-state and transient processes, and adequately describing the corresponding processes in the physical object.

DT expert models are a set of techniques, algorithms, programs, and data based on the experience of experts obtained in the design, manufacture, testing, operation, periodic and continuous diagnostics on connected and disconnected equipment, analysis of accidents of similar objects. The expert model is based on the experience of laboratory research and testing of various physical models of similar objects as a whole or their individual components and units. Expert models contain the empirical relationships between informative parameters, diagnostic criteria, and performance criteria. They also include the ranking of criteria and parameters according to the degree of the risk of deviations from their nominal values, as well as the determination of the level of acceptable values.

DT statistical models are a set of techniques, algorithms, and programs based on artificial intelligence (AI) methods that adequately describe changes in diagnostic and performance criteria depending on changes in informative parameters using machine learning algorithms and historical data on these parameters and criteria. 
The DT examination is a set of the specific information and mathematical and (or) expert and (or) statistical models that make it possible to reliably determine the current and predicted values of the corresponding specific diagnostic or performance criteria when the values of their informative parameters change. The examination can be carried out without the physical object or together with the physical object, disconnected or not disconnected from the consumer.

The Image of equipment $D T$ is a set of examinations of the DT of equipment that characterizes its functional, physical, or chemical properties and makes it possible to reliably determine the current and predicted technical condition of individual components of the physical object of the DT.

Support tools of equipment DT [5] are a set of information data, methodological, hardware, and software tools that allow you to reliably implement all images of the DT.

The digital platform of equipment DT [5] is a system of algorithmized relationships between support tools of all images of the DT, united by a single information environment.

\section{Purpose and objectives of creating the DT of equipment}

The purpose of creating DTs of any equipment is to ensure the required reliability and reduce its operating costs by making reasonable, optimal, and long-term decisions based on the use of the current and predicted values (determined using the DT) of the diagnostic criteria for the operation of individual units and components and equipment as a whole at all stages of its life cycle.

When developing a data center, it is necessary to solve a set of problems related to interdisciplinary modeling, data analysis, and machine learning. Some of these tasks are listed below:

- the selection of organizational and methodological support of the DT, including standards and methodological materials, determination of the types of users, their role and access rights, the form and volume of the information required for their work;

- the development of the DT architecture, including the development of technical requirements for all its components and images, the choice of software and hardware (digital) platforms to implement the developed architecture for collecting, transferring, storing, visualizing data, and the functioning of the software of statistical, expert and mathematical models required for the implementation of the DT of the specific equipment;

- the collection of data on the equipment, the DT of which is being developed, including manufacturer and operation data;

- the design analysis of the specific equipment, definition and description of its critical areas, description of possible defects of the equipment as a whole, its components and images, units and critical areas;

- the development of information, mathematical, expert, and statistical models to diagnose possible defects and predictions of their development, including the determination of criteria and informative parameters of physicochemical phenomena that manifest themselves in the occurrence and development of defects, methods and hardware for direct or indirect (using additional calculation) measurements of these criteria and parameters;

- the development of information, mathematical, expert, and statistical models to assess the current values of integral criteria for the technical condition of the equipment and forecasts of their development, including the asset health index (AHI), predicted service life, current and predicted load capacity;

- the analysis of the characteristics of software and hardware platforms for collecting, transferring, storing, visualizing, protecting and processing model data, including those using statistical methods of AI, expert algorithms to compare current data with acceptable values and mathematical methods for modeling electrical, electromagnetic, thermal and mechanical processes;

- the development of recommendations for clarifying the characteristics of criteria and parameters to specify the intensity and localization of defects, measures for the complete or partial elimination of defects, including preventive and repair works;

- the development of methods and data to autonomously test software and hardware components of the DT, information, mathematical, expert, and statistical models. Testing of the developed models;

- the development of methods and data to test DT software and hardware components on physical objects;

- the testing of the DT functioning when transferring data from the real physical object to the digital platform with the developed models integrated into it.

\section{DT users and their functions}

At each stage of the DT life cycle, various users perform different functions working with its data, methodological support, software, and hardware. We will conditionally divide all DT users into three types:

- Equipment Users/Customers;

- Equipment Users/Developers (including designers, buyers of materials and components, manufacturers, and testers);

- Equipment Users/Operators including installation organizations, specialists of stations and substations, specialists of power systems (quality departments and technical inspections), experts of various diagnostic centers and organizations for the corresponding equipment. In some cases, Users/Operators act as Users/Customers.

It should be noted that if the DT is developed not at the first but some other stage of the equipment life cycle, for example, at the operation stage, then it is necessary to collect in the database all the required information for the operation of mathematical, expert, and statistical models both at the appropriate stage of development and subsequent stages.

At the stage of the procurement tender: 
- Users/Customers develop technical requirements for the equipment based on regulatory and methodological documentation, studies of the features of equipment operation in specific conditions, and technical requirements for similar equipment developed by various companies. The database of the DT (at this stage - of the DTP) should contain this information and thereby improve the quality, reliability, and completeness of the technical requirements for the tender. The generated requirements are also entered into the DT database and are the first data to monitor the technical condition of equipment, including checking the developers' tender offers for compliance with the tender requirements;

-Equipment Users/Developers at this stage use the database and software of the DTP to select structure layout and carry out optimization and verification calculations of the structure proposed for participation in the tender.

At the stages of computational design and development of technological and design documentation, ordering and incoming inspection of materials and components, preparation of production, manufacturing, and testing of equipment:

- Users/Developers use the database of the DT with the specific nominal design and technological parameters of the equipment (corresponding to the drawings without taking into account manufacturing deviations) and nominal material properties (corresponding to the catalogs without taking into account their real properties as delivered). The methodological support, hardware, and software of the DTP is also used for imitating and predictive modeling of equipment in the conditions of the Developer's specific test station and specific standardized types and parameters of tests;

- based on the results of simulation and predictive modeling, decisions are made on the compliance of the design with technical requirements and a real physical object is created;

- Users/Customers control the quality of the manufacture of equipment, comparing the results of acceptance tests with the regulatory or contractual requirements. However, in many cases, the results of acceptance tests may not be enough to ensure quality control. Therefore, under contracts for the supply of equipment, Users/Customers frequently carry out quality control at all stages of equipment creation - from design to testing. To carry out inspections at these stages, data on the design of a specific piece of equipment, characteristics of materials, and parameters of its test modes are used. Moreover, to perform quality control, one needs to carry out intercomparison and analysis of deviations of the results of calculations and simulation of the DT with the results of measurements and tests of its accessories, materials, units, and components, as well as the equipment in general. This data is also required to verify that the parameters and characteristics of the equipment comply with the technical requirements of the tender and regulatory documentation. All data from the DTP base required for verification calculation and simulation, the results of these calculations and modeling, the results of measurements and tests, as well as data on the technical requirements of the tender documents, must be transferred to the Users/Customers at the appropriate stages of digital control, which makes it possible to form a database of the DT copy. At the same stage, according to the proposals of the User/Developers, the nomenclature and permissible values of the "settings" for the criteria and informative parameters of all images and examinations of the DT should be formed in the database of the twin copy. Besides, requirements are also created for the nomenclature, methods of conducting examinations for periodic and continuous monitoring of criteria, and informative parameters in operation. Sensors, instruments, devices, and systems of diagnostic monitoring and technological protection are installed on the real equipment (or some special places and control cables may be provided for installation). The characteristics and descriptions of the installation sites of this equipment are also added to the DT copy database. All specified data are used to diagnose equipment at subsequent stages of its life cycle. Besides, all information necessary not only for diagnostics but also for performing other work at subsequent stages of the equipment life cycle, including installation, operational and repair documentation, must be transmitted in digital form to the database of the DT copy.

At the stages of transportation, storage, installation, and pre-commissioning of the equipment before its commissioning, including the same work for sensors, instruments, devices and systems for diagnostic monitoring and equipment protection, users of the DT copy perform the following functions:

- Users/Developers supervise the execution of the work, controlling the quality using the information of the DT copy database;

- Users/Operators carry out works, controlling the quality using the information of the DT copy database. In addition, together with the Users/Developers, they should perform predictive modeling using mathematical and expert models of the DT and find out the effect of all changes in informative parameters on criteria changes before the actual commissioning.

At the first start-up of the equipment, the operating conditions often differ greatly from the conditions of standard tests at the manufacturing plant, e.g. different connection diagrams of the equipment, the effect of one equipment on another, currents and voltages affecting the equipment, the influence of ambient temperature and humidity, the influence of the consequences of transportation, storage, and installation. Therefore, predicting criteria values and informative parameters can significantly reduce the risks of the further operation and possible equipment failure. Based on the analysis of these forecasts, decisions on additional measures to reduce risks can be made (e.g., additional drying of insulation for power transformers). It can also be decided to replace components or units, change the schemes and start modes, etc. It should be noted that it is important to use the DT database to refine setup values and calibrating sensors, instruments, devices, and systems for monitoring and technological protection. Statistical 
algorithms that are based on machine learning methods and currently used by individual developers and manufacturers of devices let the equipment work for 1-3 months in the training mode without the control of the corresponding devices. During further operation, the calibration and setup values must still be changed, since the operating modes under nominal and starting conditions are different. In several cases, the refusal to recalibrate and refine the setup values led to errors in the operation of monitoring devices and technological protections and the occurrence of emergencies.

At the operation stages, Users/Operators can perform the following functions with the help of the DT data, software, and hardware:

- the collection, storage and visualization of the data, measured on the real object and transmitted through the substation's automated process control system (APCS) from certain devices and diagnostic monitoring systems to remote computers of users. The risk level is assessed and the presence of abnormal phenomena is revealed by comparing the current and previous values of these data with each other and comparing them with the specified permissible values. Analysis of the rate of changes of these data (trends) allows predicting the development of these abnormal phenomena in the nearest future. At the same time, it is considered that the operating conditions of the equipment and environmental conditions do not change in the forecast period. In most cases, the permissible values of the parameters do not change during the entire operation and are not determined for a specific real object but are the average values of the entire equipment population. Sensors for measuring the informative parameters of the equipment are installed not in critical areas but in other accessible places. This leads to the fact that the use of monitoring data on informative parameters of the equipment without taking into account the design data (the equipment is considered as a "black box") allows only to identify abnormal phenomena and, at the early stages of their development, to warn operating personnel about the need to take measures to reduce the risks of emergency outages. It is often not possible to identify the cause of the abnormal phenomena and to determine a specific critical area and the resulting structural defect only by using these instruments and diagnostic monitoring systems;

- the identification of the causes of abnormal phenomena in specific critical areas of the equipment using the data of diagnostic monitoring systems and the database of the design and equipment materials characteristics, the catalog of possible defects in similar equipment, mathematical, expert, and statistical models of the DT. For this purpose, simulation of criteria is performed in critical areas when changing informative parameters, as well as when changing characteristics of the environment or external influences on equipment;

-the prediction of changes in the technical condition of the equipment under planned and emergency electrical, electromagnetic, thermal, and mechanical influences, as well as changes in the physical and chemical characteristics of materials used in the equipment, due to their aging or operating conditions;
- the determination of the current and predicted values of integral indicators of the equipment, including service life, load capacity, and AHI;

- the planning of the required volume, duration, and cost of preventive and repair works.

At the operational stage, Users/Developers, together with Users/Operators (at their request), use the data and software of the DT to find out the causes of abnormal phenomena and possible defects. Based on simulation modeling and study of operating experience with the help of databases and DT models, proposals and projects for design modifications are made to increase the efficiency and reliability, as well as to extend the service life.

\section{Characteristics of the DT physical object}

The physical object, for which the DT is used for its modeling and forecasting, is the electric power equipment installed at power plants and substations. By this term, we will also mean a group of equipment for solving a common problem: electrical connection, electrical installation, station, or substation. For the DT of a group of equipment, it is necessary to develop a DT for each type of equipment included in this group. Data is collected from the physical object, including sensors, instruments, devices, diagnostic monitoring and protection systems installed on it, and is transferred to the digital platform.

Description of the data collected from physical
objects

The list of data, the method of collection and primary processing, as well as methods of data transmission and information models of data, are developed for the specific physical object. The collected data should include both data obtained automatically in a continuous mode from the APCS of the substation, equipment diagnostic monitoring systems, and data from manual instrumental control and diagnostics periodically performed on connected and disconnected equipment.

The volume of implemented information, mathematical, expert, and statistical models of the DT is determined depending on the availability, reliability, and details of the data on specific physical objects.

\section{Technical requirements for developed DTs}

To solve the above-stated tasks of the DT and ensure user functions at all stages of the life cycle, primarily operation, the images, examination, and models of the DT being developed must meet the technical requirements below.

General requirements for DT of the equipment:

- the structure of the DT should consist of images, examination, models, criteria, and informative parameters (Fig. 1); 


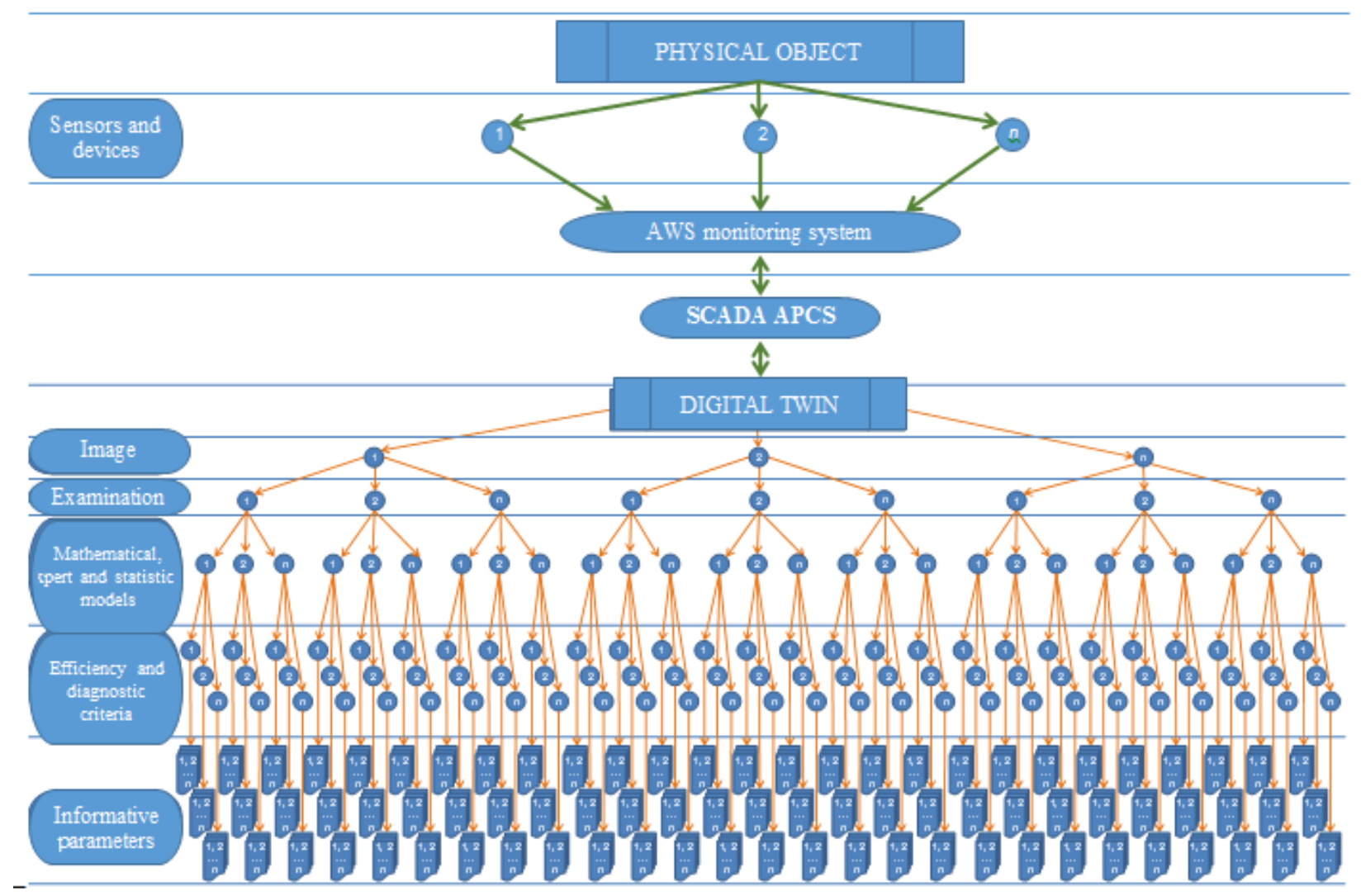

Fig. 1. Structure of equipment DT.

- geometric and structural models of the object with a description of critical areas and possible defects that are typical for the structures of specific physical objects;

- the information on technological processes of manufacturing and assembly of single elements and equipment in general;

- a set of data on impacts, criteria, parameters, accessories, components, and units of the equipment calculated at the design stage (measured at the manufacturing stage) and testing stage at the manufacturing plant, as well as on measurements and tests during installation and operation;

- informational, mathematical, expert, and statistical models describing the main physical and chemical processes occurring in the equipment and affecting its performance and service life.

The DT provides:

- the collection, primary processing, transmission of the data to a remote server, storage and processing of the data necessary for the functioning of the DT;

- self-diagnostics of measured or calculated input data, assessment of the quality and sufficiency of the data (input and output indicators) coming from monitoring objects and periodic control of the physical object;

- a real-time display of the processes taking place in the equipment; -conducting various "what-if" simulations and predictive experiments using mathematical, expert, and statistical models;

- the determination of critical areas of the equipment, current values of diagnostic and efficiency criteria, including integral criteria, forecasting their changes;

- issuing the information necessary for making management decisions, based on the results of diagnostics and modeling;

- constant "self-learning" both by changes occurring during the life cycle of the real object and the results of simulations of the conditions in which a real object was not operated.

\section{Requirements for Al mathematical, expert, and statistical models}

\section{General requirements for mathematical models \\ Mathematical models should:}

describe main physical processes occurring in the equipment under various influences and properties of the materials used (e.g., uneven distribution of the load current in certain branches of the windings and uneven distribution of voltages under different modes; the effect of the electric field on various insulation areas and determination of their electric strength reserves; the effect of magnetic fields on local and general losses of electricity in systems and structural elements; the 
influence of electrodynamic, temperature, and mechanical influences);

- determine the criteria and parameters for the conditions at all stages of the equipment life cycle;

- be parametric, for the possibility to study the influence of changes in the electrical, mechanical, and thermal external influences on the equipment technical condition;

- be parametric, for the possibility to study the effect of changes in the electrical, mechanical, and thermal characteristics of the materials used on the equipment technical condition;

- provide the ability to predict the values of the output parameters by changing the input parameters for a given time;

- at the user request, submit reports with the current and predicted output and calculation-diagnostic data;

- visualize the current and predicted output and calculated-diagnostic data in a user-friendly form for analysis and decision-making;

- be tested by their successful application in the design, manufacture, and testing of the equipment during its operation for at least 3 years.

Requirements for expert models should:

- classify the calculated or measured parameters of the object as informative or non-informative to identify possible defects, and predict the values of criteria and parameters characterizing a specific defect with a certain possibility using empirical relationships or growth and decline trends of their change;

- be confirmed by the results of examinations of physical objects similar to the real equipment in case of accidents or inconsistencies identified during operation or at the manufacturing plant. In some cases, they can be confirmed by the results of special tests on physical models or by laboratory studies. The experience in using expert models for similar structures should remain successful for at least 3 years.

\section{Requirements for AI statistical models}

Statistical AI models must meet the following requirements:

- to automatically search for the relationship between the input informative parameters and the output criteria for each defect and characteristics of the object materials specified in the expert models. Relationship search should be done, for example, by using machine learning methods and mathematical statistics. Models must be capable of visualization and manual adjustment of relationships;

- to calculate the criteria for the found relationships and compare them with the results of determining the criteria for mathematical and empirical models, if such criteria are determined by these models. The compared values are used for verification, validation, and machine learning of statistical models together with the data from direct measurements of informative and diagnostic parameters;

- to ensure the compilation of the "pattern library" of the input parameters: identification of possible variants of changes in the input parameters based on the retrospective data, the possibility of manual editing of patterns of the input parameters, the ability to set disturbances for the input parameters;

to predict the output parameters based on the "trained" models, predicted input parameters, the pattern library of the input parameters, and manually specified disturbances.

\section{DT architecture requirements}

The DT must be able to work with large amounts of big data (processing terabytes of data per day). At the same time, it should contain all images that describe changes in the condition of the real equipment under all its current and predicted modes, including those that have not occurred yet. In the course of the DT operation, it is necessary to implement data caching at all levels of information processing to prevent the repeated launch of the same queries by different users.

Only the Customer or a specialized expert organization should have access to DT hardware and software that remotely receive data from the physical object using secure communication channels. They should be based on an instrumental platform that provides data reception, storage, and processing following Russian and international standards. To implement information models, the DT platform must contain tools for providing remote user access to the database and user-friendly data visualization.

To implement mathematical, expert, and statistical DT models, multiphysics modeling (such as ANSYS or COMSOL) and AI tools (such as PYTHON) must be integrated into the platform. The integration of information, mathematical, expert, and statistical models involves the development of special software gateways for transforming data used by various applied model programs, multiphysics modeling and AI tools.

\section{Requirements for DT images}

To ensure correspondence of the DT to the real physical object and to perform user functions (Developer, Customer, and Operation), the DT must contain a sufficient number of images. In general, for electric power equipment it is necessary to implement the following 11 images: "Virtual structure", "History of events", "Physicochemical", "Visual", "Electrophysical", "Electromagnetic", "Electrodynamic", "Thermal", "Mechanical", "Acoustic" and "Technical and economic".

It should be noted that for a sufficient correspondence of the image to the real object, each image must contain at least three different examinations for each diagnostic and efficiency criterion.

\section{Requirements for DT functioning modes}

In general, it is necessary to provide the possibility of DT functioning in the following modes:

- main mode, when DT components work properly; 
- mode of testing and other technical work, in which it is allowed to carry out technological work on certain components while ensuring the full DT availability;

- emergency mode, in which the DT availability is reduced until the emergency is eliminated.

In conclusion, we would like to note that this work contains only the main requirements that are of fundamental importance for the development of the DT of the equipment. Some requirements, for example, reliability, safety, number and qualification of personnel, protection of information from unauthorized access, information safety in case of accidents, and some others, are not discussed in this work since they are well developed and are given in the relevant regulatory and technical documentation.

\section{Conclusions}

1. Proposed terms and definitions allow to unambiguously interpret the technical requirements for the DT and to describe its structure.

2. The goals and objectives of the development of electric power equipment DTs are established.

3. The functions of DT users at all stages of the life cycle of electric power equipment are given.

4. The basic principles of developing the architecture of DTs in the form of images and examinations and the functions of their components are given.

5. The general technical requirements for DTs of electric power equipment at all stages of its life cycle are proposed.

\section{References}

1. Digital twins and simulations, ABB Review, 2 (2019)

2. Q. Qi, F. Tao, Digital Twin and Big Data Towards Smart Manufacturing and Industry 4.0: 360 Degree Comparison, IEEE Access, 6 (2018)

3. F. Tao, F. Sui, A. Liu, Q. Qi, M. Zhang, B. Song, Z. Guo, S.C.-Y. Lu, A.Y.C. Nee, Digital twindriven product design framework, IJPR, 57 (12) (2019)

4. Digital twins of industrial equipment and technological processes, Digital transformation factory (2019) (in Russian)

5. Digital twins in the high-tech industry, Expert and analytical report, Moscow, Technet (2019) (in Russian)

6. A. Prokhorov, Digital twins, Concept is evolving (2018) (in Russian)

7. M. Lipatov, The first Russian predictive analytics complex for power and industrial equipment, Oil Gas Exp., 3 (49), 82-83 (2016) (in Russian)

8. S. Yeroshenko, A. Khalyasmaya, Digital Twin Technologies in Power Engineering, Proc. of Jub. Xth Int. Res. Tech. Conf. P. Eng. through the Eyes of Youth, 55-58 (2019) (in Russian). 